\title{
La reforma del sector sanitario en Cataluña en los últimos veinte años. El caso de los servicios sanitarios hospitalarios de la Generalitat de Catalunya
}

\author{
Inma Pastor Gosálbez \\ Universidad Rovira i Virgili \\ inma.pastor@urv.cat
}

\section{Resumen}

Los objetivos de este artículo son analizar el contenido y los resultados de la lógica gerencialista o neoempresarial que se está implantando en los últimos años, de manera general, en las Administraciones públicas. Con este artículo se pretende aportar evidencias empíricas sobre los efectos de la aplicación de esta lógica empresarial en las formas y condiciones de trabajo, para, de este modo, discutir la inevitabilidad de este proceso gerencialista en la búsqueda de mayor eficiencia y eficacia. También se ba querido poner de manifiesto cuál es la especificidad de la Administración Pública en relación con la gestión de los trabajadores y de sus condiciones de trabajo.

Palabras clave: gerencialismo, administración pública, condiciones de trabajo.

\begin{abstract}
The objectives of this article proceed along the way of analysing the contents and outcomes of the managerial or neoentrepreneurial logic that is becoming established, in a general way, in public Administrations. This article tries to bring empirical evidences on the effects of this managerial logic in the forms and conditions of work and, in this way, to discuss the inevitability of this process in the quest for major efficiency and efficacy of the public Administration.
\end{abstract}

Key-words: managerialism, public administration, working conditions. 


\section{Introducciónः presentación y objetivos}

El hecho contextual que guía este artículo ${ }^{1}$ es que en la Administración Pública se está aplicando un programa de actuación basado en el modelo de las empresas privadas que identificamos con la denominación de gerencialismo. En la literatura managerial sobre el gerencialismo, éste se entiende como una propuesta de carácter meramente técnico (una nueva reedición del one best way, aunque con características no estrictamente tayloristas) que conduce a la mejora sustancial de la eficacia y la eficiencia de las organizaciones. Se trata de la importación, desde el mundo privado al mundo público, de técnicas de gestión que introducen racionalidad en la Administración Pública a través de mecanismos que se presentan sobradamente contrastados (ya que el supuesto de partida es que la gestión privada siempre es superior a la gestión pública). Sin embargo, el estudio de la aplicación de las técnicas gerenciales en la Administración Pública, desde el punto de vista de la sociología del trabajo, nos introduce en un camino aún por explorar, cuyo punto de partida es el cuestionamiento de dicha superioridad técnica, en tanto que las prácticas gerenciales no pueden separarse de las implicaciones que tienen sobre los procedimientos de trabajo y sobre las condiciones de la prestación del trabajo.

El objetivo de este artículo no es únicamente describir la forma y las características de la gestión de los trabajadores. El enfoque sociológico que aplicamos busca ir más allá y discutir la aplicación de las técnicas de gestión privadas en el contexto de las organizaciones públicas. Es decir, lo que pretendemos es, por una parte, poner de manifiesto los resultados de estas formas de gestión inspiradas en el modelo privado con el fin de conocer el papel que desempeñan los actores en su definición y los efectos que tienen sobre las condiciones de trabajo y, por otra parte, contrastar si estas prácticas del gerencialismo privado introducidas en las organizaciones públicas generan realmente, de acuerdo con la justificación de su implantación, servicios más eficientes, de más calidad y más en consonancia con las demandas de los usuarios.

Con este objetivo, se ha realizado un análisis comparativo del modelo de gestión privado y del modelo de gestión pública aplicados en el Departamento de Salud de la Generalitat de Catalunya. Como lo que nos interesa es la comparación entre sector público y sector privado, los criterios de selección de los casos estudiados se han basado en dos variables consideradas claves: la propiedad pública o privada del hospital y el tipo de gestión que se practica. De esta forma diferenciamos la gestión que se hace desde la aplicación del derecho administrativo de la gestión que se hace desde la aplicación del derecho privado. Del cruce de estas dos variables resulta una tabla de

1 Este artículo presenta de forma resumida el análisis realizado por la autora en su tesis doctoral. Dicha tesis mereció el premio del Consejo Económico y Social de España a tesis doctorales en la convocatoria del año 2005. 
doble entrada con cuatro categorías diferentes (cuadro 1). Sólo tres de estas categorías pueden identificarse dentro de los servicios sanitarios hospitalarios que ofrece el Departamento de Salud de la Generalitat de Catalunya: los hospitales de propiedad y gestión pública, los hospitales de propiedad pública y gestión privada y los hospitales de propiedad y gestión privada. En el trabajo de campo que fundamenta este artículo se han realizado sendos estudios de caso de tres hospitales, uno por cada uno de los tres tipos considerados.

Cuadro 1. Tipos de organizaciones presentes en los servicios sanitarios según el tipo de propiedad y la forma de gestión

\begin{tabular}{|l|l|l|l|}
\cline { 3 - 4 } \multicolumn{2}{c|}{} & \multicolumn{2}{c|}{ Tipo de propiedad } \\
\cline { 3 - 4 } \multicolumn{2}{c|}{ Forma de gestión } & Pública & Privada \\
\cline { 2 - 4 } & Privada & Caso 1 & - \\
\hline
\end{tabular}

Lo que queremos saber, a partir de este estudio, es en qué ha consistido la introducción de las prácticas gerenciales en el funcionamiento de los hospitales que prestan servicios en el ámbito público. Asimismo, queremos conocer los efectos que estas prácticas tienen sobre las condiciones de trabajo, en la medida en que el gerencialismo también tiene consecuencias sobre la organización del trabajo (en el sentido de cómo se organizan y gestionan los servicios), sobre el modus operandi de la prestación de los trabajadores (cómo influye en las condiciones del trabajo individual y colectivo) y, finalmente, sobre las relaciones sociales existentes en el centro de trabajo (capacidad de negociación de los actores, individualización de la relación laboral, participación de los actores en el diseño y la carga de trabajo...). Este análisis se desarrolla en torno a dos grandes ámbitos temáticos: el primero hace referencia al proceso de gerencialización y a las innovaciones que se están dando en la manera de gestionar los hospitales catalanes; el segundo ámbito analiza los efectos que la introducción de herramientas gerenciales está teniendo sobre la condiciones de trabajo en los hospitales.

Para situar el análisis, empezamos por exponer el marco general de funcionamiento del sistema sanitario y sus características en el caso de Cataluña.

\section{Características y funcionamiento del sistema sanitario en Cataluña}

A finales de la década de 1970, cuando el Estado del bienestar es cuestionado tanto por algunos discursos ideológicos como por acciones políticas concretas, se inicia de 
nuevo una reconfiguración del aparato estatal. En este escenario, lo público deja de ser un monopolio estatal para adoptar una forma nueva: un espacio para el conjunto de individuos, agencias y agentes de una sociedad, dentro del cual las dependencias gubernamentales son solamente uno de los componentes. El Estado se compromete en la articulación de los esfuerzos del conjunto de los actores, al tiempo que deja a un lado su protagonismo exclusivo en la atención de los asuntos públicos.

Según Cabrero Mendoza (1998), a la vista de esta situación, hay que entender, analizar y describir un sistema de redes de dependencias gubernamentales más que un aparato gubernamental, concepto cada vez menos útil en el estudio de la acción de gobierno. Las redes de dependencias, si bien todas ellas gubernamentales, compiten, se disputan espacios y recursos, desarrollan lógicas sólo parcialmente comunes y, en cambio, pueden mostrar grandes diferencias. Es en este momento cuando la toma de decisiones, la visión estratégica, el juego de actores, los problemas de eficiencia, las preferencias cliente-usuario, entre otros aspectos, surgen como elementos fundamentales del análisis. Un escenario como el descrito es un campo propicio para la aparición de la gestión y la gerencia pública. ${ }^{2}$

Además, en los últimos se han desarrollado una serie de reformas políticas ${ }^{3}$ que ponen especial énfasis en la reestructuración de los objetivos y los principios básicos del propio sistema de atención sanitaria, así como en las adaptaciones administrativas del sistema existente, preservando las tradiciones históricas y los valores culturales. A continuación exponemos los cambios que se han dado en la prestación de los servicios sanitarios en el caso de la Administración Pública de Cataluña; en especial, analizaremos el caso de los servicios hospitalarios. El período temporal de este estudio abarca hasta el año 2004; se incluyen, pues, las políticas desarrolladas por diferentes gobiernos.

La reforma de la Administración sanitaria catalana es parte de una tendencia internacional de reforma ${ }^{4}$ del sector público en la dirección marcada por la denominada nueva gestión pública (new public management, NGP). El cambio fundamental derivado de estas reformas en numerosos países de la OCDE consiste en pasar de un modelo organizativo jerárquicamente integrado de financiación y provisión de servicios, a un modelo de relaciones contractuales entre estructuras desagregadas o, como apunta el gerente entrevistado del hospital del ICS, un "modelo en red entre todas las entidades que prestan finalmente el servicio sanitario; aunque la mayor importancia la tenga la propia prestación facultativa dentro, por ejemplo, del Hospital, estas actividades facultativas no se podrían realizar con un mínimo de garantías sin otros servicios colaterales

\footnotetext{
2 Según Bozeman (1998), los orígenes de la gestión pública quedan oscurecidos por el hecho de que esta disciplina es producto de la evolución institucional más que del desarrollo intelectual.

3 Según Albizu y Olazarán (2003), estas reformas están muy relacionadas con los elevados costes de los servicios sanitarios.

4 Ver Gallego Calderón (1996).
} 
y de apoyo importantes. Las empresas que prestan estos servicios forman una red de empresas que interactúan bajo los requerimientos del hospital».

Aprovechando la flexibilidad de la Ley General de Sanidad (LGS) de 1986, de aplicación estatal, se constituyó el denominado Servicio Público de Salud en Cataluña a través de una adaptación de la legislación general del Estado a los hechos históricos e idiosincrásicos de la realidad catalana, es decir, teniendo en cuenta básicamente la distribución territorial de los recursos sanitarios, así como las fórmulas de gestión desarrolladas en este territorio. El modelo sanitario catalán es un modelo sanitario mixto, que integra en una sola red de utilización pública todos los recursos sanitarios, sean o no de titularidad pública, y que recoge una tradición de entidades (mutuas, fundaciones, consorcios, centros de la Iglesia) históricamente dedicadas a la atención de la salud.

A partir de 1981, el Gobierno de la Generalitat se planteó la disyuntiva entre la ampliación de la red propia de la Seguridad Social o la potenciación de estructuras de servicios ajenas a la Seguridad Social, manteniendo un conjunto de centros propios dentro de la estructura del Departamento de Sanidad y Seguridad Social ${ }^{5}$ a través de la figura del ICS. Finalmente, se tomó este segundo camino como vía de desarrollo para el nuevo servicio sanitario catalán, en consonancia con los planteamientos políticos del partido político que gobernaba en ese momento en Cataluña. De este modo, fundaciones privadas, órdenes religiosas, administraciones locales, mutualidades de previsión, etc., propietarios de centros y servicios sanitarios, quedaron integrados en la denominada Red Hospitalaria de Utilización Pública (XHUP, en sus siglas en catalán). Así pues, aprovechando las posibilidades de la LGS, su desarrollo en Cataluña adoptó el modelo de gestión diversificada de los servicios sanitarios. Finalmente, la Ley de Ordenación Sanitaria de Cataluña (LOSC) de 1990 validó normativamente el modelo de separación de funciones, modelo que adoptó la forma que se detalla en el cuadro 2.

\section{Cuadro 2. Modelo de separación de funciones sancionado por la LOSC}

\footnotetext{
Planificación y financiación: Departamento de Sanidad y Seguridad Social de la Generalitat de Catalunya.

Aseguramiento y compra de servicios: Servicio Catalán de la Salud.

Provisión de servicios: entidades prestadoras de titularidad pública o privada. La prestación puede ser directa (mediante el ICS), indirecta (mediante entidades ajenas) o compartida (mediante consorcios con participación pública y privada).
}

Fuente: elaboración propia a partir del texto de la Ley de Ordenación Sanitaria de Cataluña (LOSC) de 1990.

5 Desde mayo de 2004, este departamento ha pasado a denominarse Departamento de Salud. Empleamos las dos denominaciones porque a veces se hace referencia al Departamento en una época anterior, en la que su nombre no era el que se emplea ahora. 
Figura 1

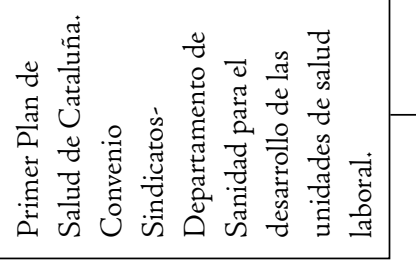

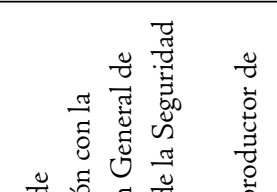

यू

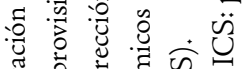

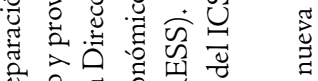

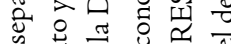

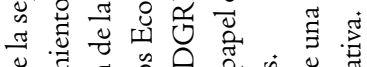

ช चี

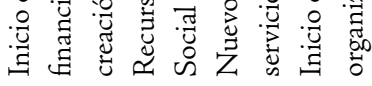

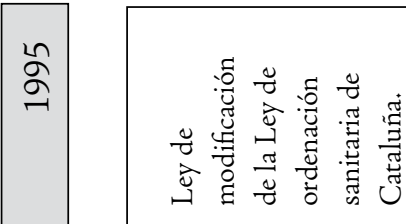

๙

๙ू

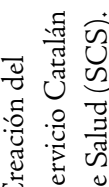

نे ญ्ष

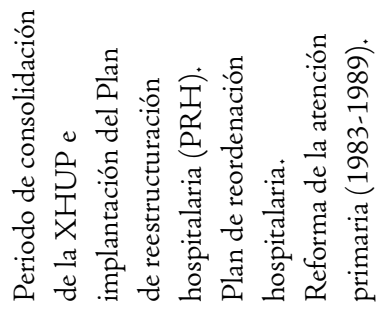

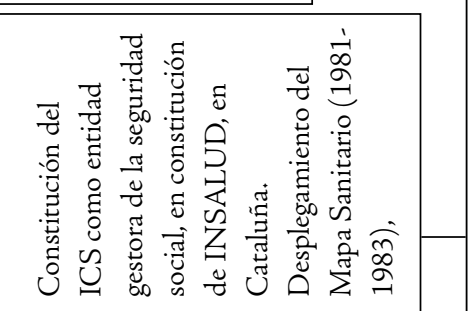

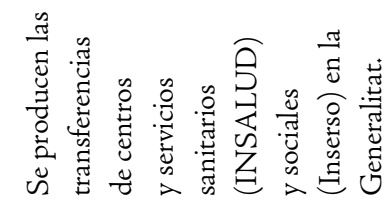

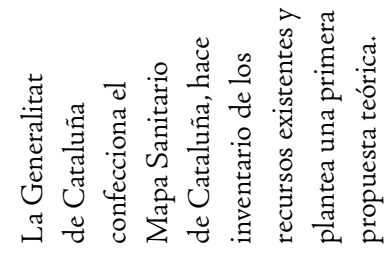
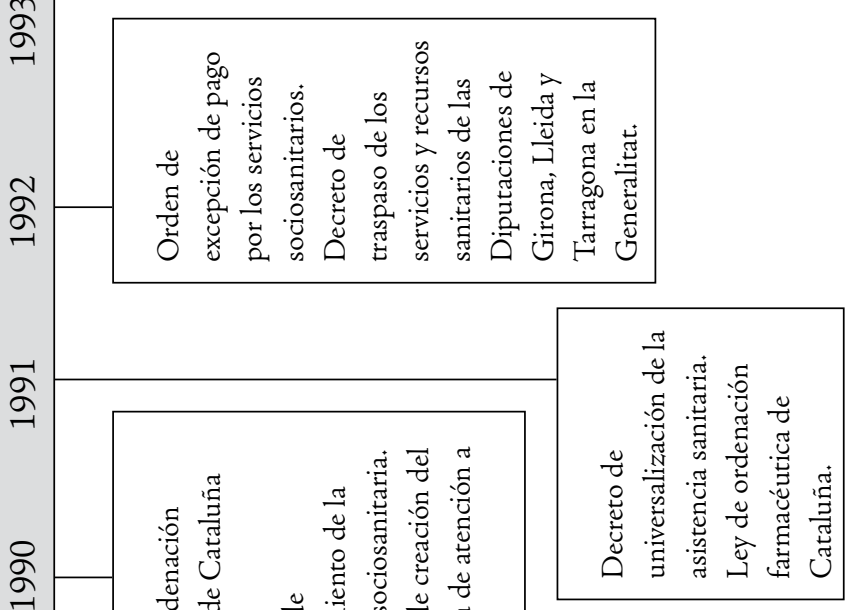

苛

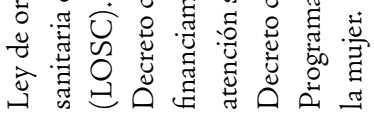
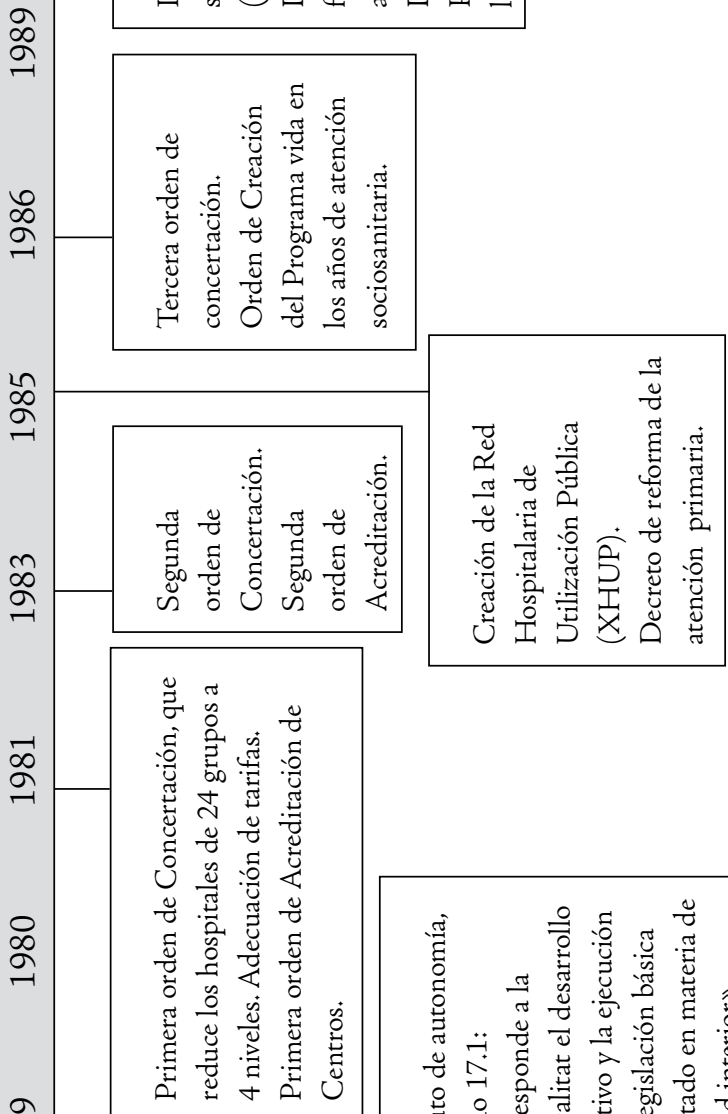

ลิ

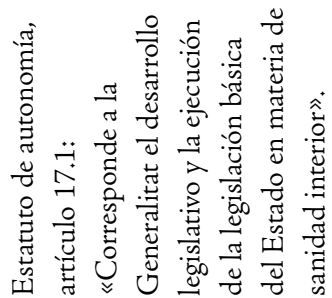




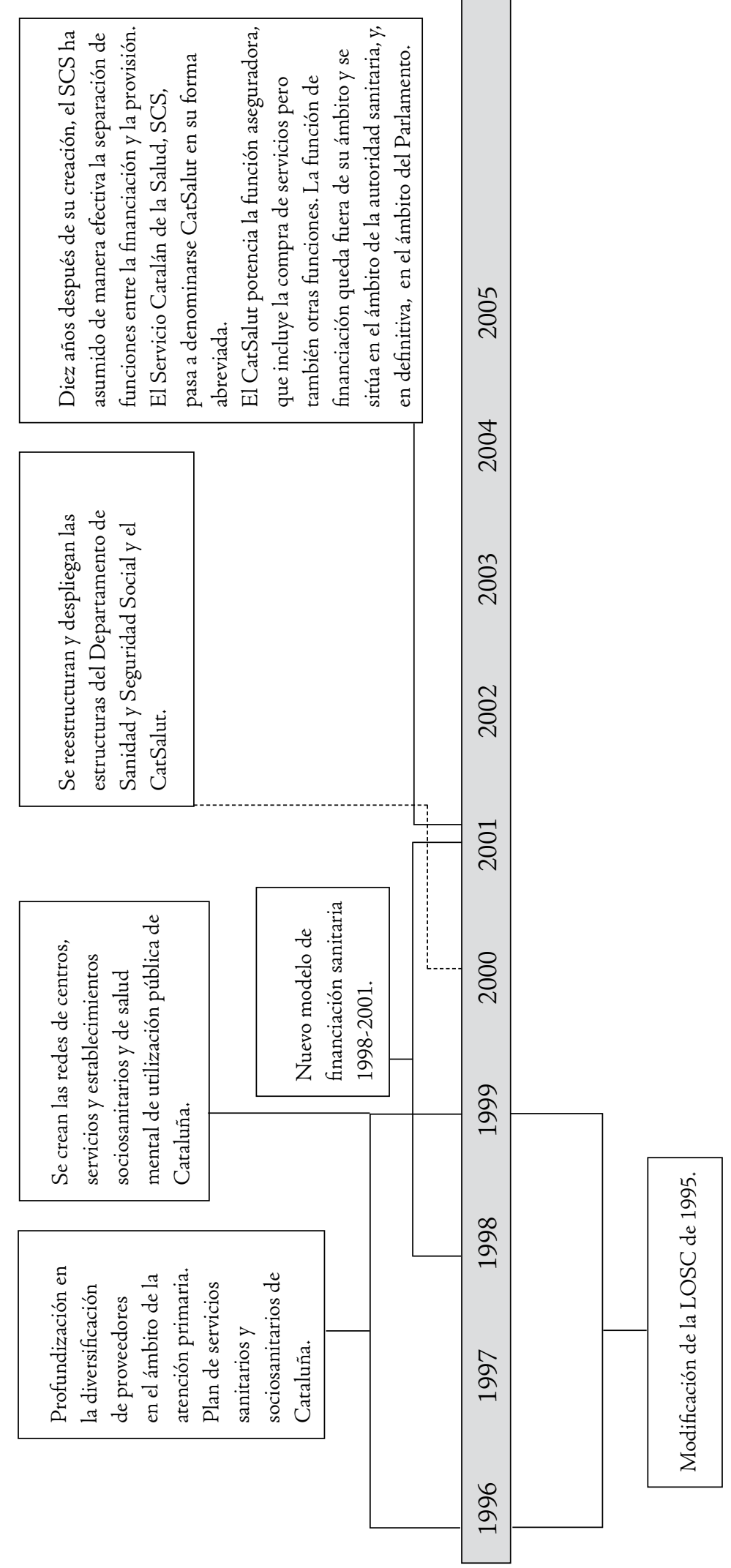


A diferencia de este modelo, el del INSALUD 6 financiaba, aseguraba y prestaba los servicios directamente. El modelo catalán está resultando, para algunos analistas - entre ellos los sindicatos-, más caro en su funcionamiento que el modelo integrado, ya sea por los costes de transacción del sistema de concertación, ya sea por la implantación de la red comarcal de servicios existentes, además del coste del Plan de Reordenación Hospitalaria, que fue necesario para adecuar la red hospitalaria de propiedad ajena a la Seguridad Social, para que pudiera alcanzar los niveles exigidos de acreditación. Presentamos de forma esquemática el cronograma de los principales hechos legislativos que dieron paso a la gestación del actual sistema sanitario catalán (ver Figura 1).

En 1980, la Generalitat de Catalunya presenta una primera propuesta teórica de ordenación del sistema sociosanitario catalán, y al año siguiente se producen las transferencias del INSALUD a la Generalitat en un contexto caracterizado por la desorientación y el desorden dentro del propio sector, fruto de la falta de planificación, por la insuficiencia histórica del desarrollo de la red de seguridad social, la descapitalización por falta de inversiones, los grandes desequilibrios territoriales y tecnológicos y un sistema de concertación del INSALUD nada conveniente a las necesidades reales. El mismo año de 1981 se promulgaba la primera orden de acreditación de centros, ${ }^{7}$ que conllevaba la revisión externa de la estructura y la garantía de unos mínimos estándares de calidad y establecía la vinculación con la concertación. Dos años más tarde, en 1983, se llevaba a cabo el despliegue del mapa sanitario, que consistía en el desarrollo del sistema hospitalario, el aprovechamiento de los recursos hospitalarios con independencia de su titularidad, el redimensionamiento de la red y la potenciación de los hospitales comarcales (los generales básicos).

En 1985 se crea la Red Hospitalaria de Utilización Pública (XHUP) como dispositivo estable de asistencia hospitalaria de financiación pública, sometida a la planificación, compuesta por centros de diferente titularidad y con requerimientos establecidos de incorporación.

La Ley de Ordenación Sanitaria de Cataluña (LOSC) permitirá en el año 1990 la consolidación de la XHUP y abrirá el camino para avanzar en las nuevas modalidades de gestión: directa, indirecta y compartida. En la exposición de motivos de la LOSC se hablaba de la necesidad de separar la responsabilidad de planificar y asegurar la cobertura sanitaria eficiente y eficaz a partir de los recursos disponibles, de la responsabilidad de gestionar la provisión de los servicios sanitarios. Entre otros objetivos, constaba el objetivo explícito de evitar las rigideces derivadas de la estructura y naturaleza jurídica del Instituto Catalán de la Salud (ICS). Se proponía hacer realidad este objetivo

\footnotetext{
6 Organismo estatal de gestión de los centros sanitarios de propiedad pública.

7 La acreditación es un proceso de evaluación externa de centros sanitarios que nació como iniciativa promovida por el propio sector sanitario en Estados Unidos en 1919. Posteriormente se extendió a otros países. Cataluña fue pionera en Europa, ya que fue la primera en crear, en 1981, un programa de acreditación.
} 
a través de la creación de un nuevo ente público de carácter institucional adscrito al Departamento de Sanidad y Seguridad Social (DSSS), el Servicio Catalán de la Salud (a partir de ahora, CatSalut ${ }^{8}$ ), creado finalmente en 1991. El cuadro 3 lo resume.

\section{Cuadro 3. Rasgos generales de la LOSC (1990)}

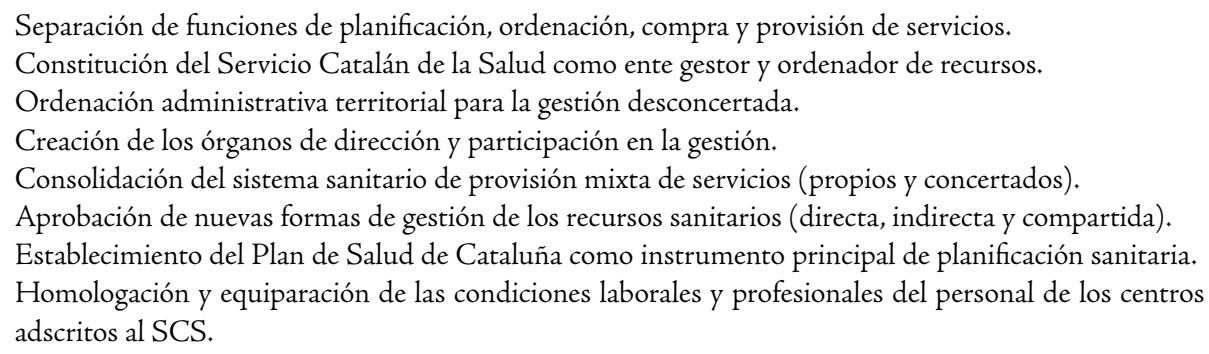

Fuente: elaboración propia a partir del texto de la Ley de Ordenación Sanitaria de Cataluña (LOSC) de 1990.

El CatSalut nace con la responsabilidad de proveer servicios sanitarios a la población con total libertad para recurrir a fórmulas de gestión tanto públicas como privadas, de manera que sólo las funciones de regulación y financiación del servicio público de salud quedan sujetas al derecho público. La libertad de recurrir a fórmulas diversas implica una gestión indirecta de los recursos que incluye las siguientes posibilidades: la creación de nuevos proveedores - empresas públicas - el establecimiento de acuerdos para una gestión integrada, la creación de consorcios públicos que puedan crear organismos de carácter institucional y la creación o participación en otras organizaciones públicas o privadas. Actualmente, el CatSalut se define a sí mismo como la aseguradora pública de Cataluña, ya que actúa como garante de las prestaciones sanitarias de cobertura pública. El CatSalut ${ }^{9}$ planifica, compra y evalúa los servicios sanitarios en función de las necesidades de la población desarrollando, por una parte, una gestión integral de la oferta y la demanda y, por otra, la comunicación con los ciudadanos y su participación. De este modo, la descentralización y la desconcentración de la gestión son principios centrales de la LOSC.

A partir de la LOSC, el CatSalut se convierte en una organización nueva y asume las funciones del ICS, que deja de ser una organización gestora de los servicios sani-

8 Sin embargo, esta denominación, la de Catsalut, no se implanta hasta 2001. Hasta ese año, los textos y documentos oficiales denominaban al Servicio Catalán de la Salud con las siglas SCS.

9 Más claramente, a partir del año 2001, el CatSalut potencia la función aseguradora, que incluye la compra de servicios, pero también otras funciones. La función de financiación queda fuera de su ámbito y se sitúa en el ámbito de la autoridad sanitaria y, en definitiva, en el ámbito del Parlamento. 
tarios de la Seguridad Social para convertirse, en la práctica, en un gestor y proveedor competitivo, con el resto de los proveedores, de servicios sanitarios públicos. Lo ilustra la figura 2 .

Figura 2. Esquema de la relación entre proveedores de la red hospitalaria y el Departamento de Salud

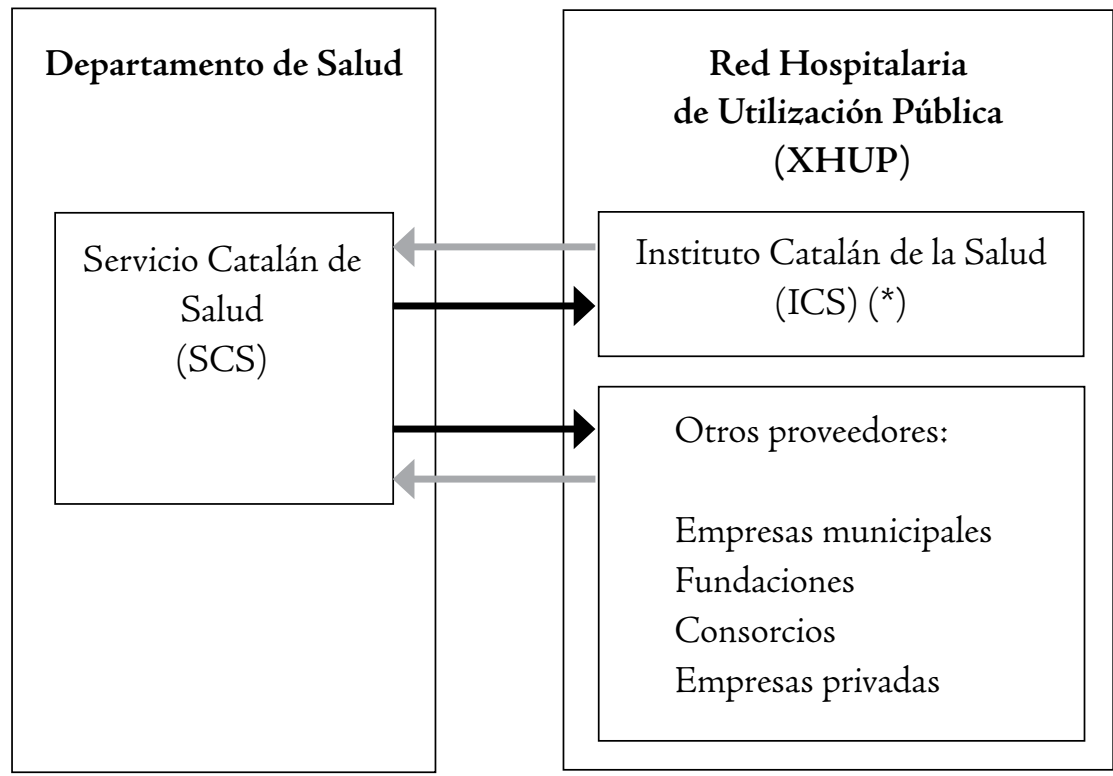

$\longrightarrow$ Venta de servicios

Compra de servicios

${ }^{*}$ ) El Instituto Catalán de la Salud pertenece, orgánicamente, al Departamento de la Salud (como queda reflejado en la figura 1), pero funcionalmente forma parte de la XHUP como proveedor público de servicios hospitalarios.

Según apunta Gallego (1996), la base para la separación entre los roles de comprador y proveedor de servicios sanitarios fue un informe elaborado por la consultora McKinsey\&Company en el año 1991 a petición del Departamento de Sanidad y Seguridad Social. De hecho, este informe sirvió para interpretar el nuevo modelo sanitario catalán. Además de la separación, ya comentada, entre comprador y proveedor, las recomendaciones del informe fueron la necesidad de introducir un estilo de gestión propio del sector privado en ambas funciones 
(comprador y proveedor). Se afirmaba en aquel informe que la competencia gestionada facilitaría la consecución de los objetivos de eficiencia y eficacia, así como el objetivo de unos servicios de calidad orientados al consumidor, objetivos que la misma LOSC especifica.

Es así como se propone que el CatSalut adopte el rol de comprador de servicios, lo que implica responsabilidades tales como definir y evaluar las necesidades sanitarias, es decir, planificar y asegurar la cobertura de estas necesidades mediante la asignación de recursos por medio de contratos con los proveedores. Esto requiere que el CatSalut establezca una serie de objetivos y de indicadores de resultados para supervisar el cumplimiento de los contratos $y$, de este modo, evaluar la eficiencia y la calidad de los servicios provistos. De esta manera, el CatSalut no sería responsable de la ineficiencia de los proveedores, sino de errores en la planificación y gestión de la competencia a través de incentivos contractuales. En este modelo, los proveedores deben competir por los contratos del CatSalut según la eficiencia y la calidad de sus servicios. Según las recomendaciones del informe, la diversidad de proveedores debería ser impulsada también en la asistencia primaria; de esta manera se rompe el monopolio que en este ámbito tiene (tenía) el ICS. Igualmente, el informe recomienda que los proveedores adopten un estilo más empresarial y competitivo y que desarrollen nuevos sistemas de información y gestión y transformen su cultura organizativa.

Según el CatSalut, las actividades de evaluación, control y mejora de la calidad son un elemento fundamental en el proceso para la contratación de la gestión de servicios sanitarios. Por eso se ha establecido un nuevo marco de referencia por lo que respecta a los requerimientos de información que debe presentar cada tipología de centros. El Decreto 169/1996, de 23 de mayo, exige como requisito para la formalización de los convenios y los contratos de gestión de servicios sanitarios que los centros cumplan los estándares de calidad que se determinen para cada tipología de centros. Los tres aspectos básicos a los que hacen referencia estos estándares son:

1. Aspectos organizativos y de personal, que valoran el sistema organizativo del centro y el funcionamiento del servicio, así como los recursos humanos, las funciones que desarrolla el personal y la formación de éste.

2. Aspectos de calidad de la asistencia sanitaria y de la atención global al usuario, que incluyen los sistemas de recogida de datos y los circuitos de acceso y de seguimiento, por una parte, y atención y estímulo a la participación del ciudadano, por otra. 
3. Aspectos relativos al control y los protocolos de mantenimiento de la estructura, las instalaciones y los equipamientos del centro.

En la práctica, siguiendo las recomendaciones apuntadas, el CatSalut ha adoptado un rol de comprador de servicios sanitarios. La idea que articula todo este proceso es la necesidad de que el CatSalut gestione la demanda, es decir, de que la planificación se base en la evaluación de necesidades. Esta planificación se hará con un instrumento base, el Plan de Salud de Cataluña. Los contratos con los proveedores se establecerán a partir de las directrices que marque este Plan. Un tercer elemento ha sido sobradamente utilizado por el CatSalut en la gestión de los proveedores: la creación de empresas públicas (con un amplio margen de maniobra dentro del derecho privado) adscritas a actuar como proveedores directos del CatSalut. ${ }^{10}$

Por su parte, el ICS ha experimentado un proceso de reforma dirigido a convertirlo, exclusivamente, en proveedor del CatSalut. El punto de partida del ICS era tal que su situación se caracterizaba, a principios de los años noventa, según informes del propio ICS (ICS, 1994), como una organización en la que no había espíritu de dirección ni de grupo; la calidad casi no se medía, y el enfoque presupuestario eludía medidas de eficiencia y eficacia; no existía cultura de provisión; no había gestión de carreras profesionales... A partir de aquel momento se pusieron en marcha tres líneas de reforma que se han aplicado al ICS a lo largo de los años noventa. En primer lugar, la estructura organizativa se basa no en criterios organizativos, sino en líneas de servicios (centro corporativo, hospitales y asistencia primaria). En segundo lugar, se promueve la participación de los profesionales y su responsabilidad. En tercer lugar, se introducen sistemas de gestión tales como las auditorías externas, la contabilidad, los controles financieros y la facturación. ${ }^{11}$

Finalmente, en 1995, se lleva a cabo una modificación de la LOSC con la introducción de nuevas formas de gestión cooperativa, con ánimo de lucro, y la posibilidad de transformación de la naturaleza jurídica del Instituto Catalán de la Salud y la creación del Consorcio Sanitario de Barcelona.

Esta ley de modificaciones apunta de forma explícita al hecho de que uno de los objetivos que se pretende es la ruptura del monopolio del ICS en la provisión de atención primaria. De aquí que la ley prevea promover la creación de entidades a partir de equipos de profesionales para optar a contratos directos con el CatSalut como provee-

10 Como ejemplos se pueden citar el Instituto de Diagnóstico por la Imagen; la Energética de Instalaciones Sanitarias, S.A.; el Sistema de Emergencias Médicas, S. A.; la Agencia de Evaluación de Tecnología e Investigación Médicas; el Instituto Catalán de Oncología, y los Servicios Sanitarios de Referencia (Centro de Transfusión y Banco de Tejidos, entre otros).

11 A lo largo de 1992 y 1993, la asignación presupuestaria anual del ICS fue traducida a términos contractuales por primera vez, de manera que los hospitales tuvieron que empezar a facturar para que las asignaciones presupuestarias del ICS estuvieran relacionadas con los costes de cada actividad. 
dores privados. Éste es un proceso que implica una transformación progresiva de las condiciones de trabajo y las relaciones laborales en el sector sanitario, ya que supone el paso de un marco de regulación estatutario a otro contractual. En opinión de los sindicatos, la Ley de modificación de la LOSC profundiza en la vía de la privatización de la gestión ajena y en la empresarización de la gestión propia.

\section{Análisis}

La aplicación de reformas en el sector público desde la orientación de la nueva gestión pública implica fundamentalmente, según Dunleavy y Hood (1994), cambios en dos coordenadas: por una parte, la reducción del grado en que las normas procedimentales limitan la discrecionalidad de actuación, el denominado control de la discrecionalidad; por otra, la atenuación de la separación y diferenciación del sector público con respecto al privado. La primera coordenada es el resultado de dar mayor poder de decisión y actuación a los gestores y de la generalización de medidas de actividades, así como del control basado en resultados. La segunda coordenada se aplica en la medida en que se desagregan estructuras hasta ahora integradas, se promueve la competencia entre unidades autónomas públicas y privadas y se propone la adopción del estilo de gestión propio del sector privado.

Según Gallego (1996), estas coordenadas podrían corresponder a la distinción que hace Pollit (1993) entre las reformas administrativas calificadas como neotayloristas de los años ochenta y la aplicación de la NGP de los años noventa. En la medida en que las reformas de los años ochenta se caracterizaron por su énfasis en el control y la supervisión exhaustivos, la reducción de costes, la eficiencia, la economía, los indicadores de actividades y la remuneración en función de resultados representarían el énfasis en la primera dimensión, la discrecionalidad, ya que representan una reducción del grado en que las normas procedimentales limitan la discrecionalidad de actuación. Esta primera dimensión también ha sido denominada incentivación. En cambio, a partir de los años noventa, el énfasis se habría puesto en la calidad, la eficacia y la orientación al consumidor. Lo que permite identificar la segunda dimensión, la desagregación, no son estos valores en sí, sino las reformas estructurales que los han acompañado y que han contribuido a borrar las fronteras entre el sector público y el privado. Lo presentamos en el cuadro 4. 


\section{Cuadro 4. Componentes de la NGP}

\begin{tabular}{|l|l|}
\hline $\begin{array}{l}\text { Dimensión 1: } \\
\text { Limitación de la discrecionalidad }\end{array}$ & $\begin{array}{l}\text { Dimensión 2: } \\
\text { Menor diferenciación de los sectores públicos y } \\
\text { privados }\end{array}$ \\
\hline $\begin{array}{l}\text { Discrecionalidad para los gestores } \\
\text { Estándares de medida de actividad } \\
\text { Control de resultados }\end{array}$ & $\begin{array}{l}\text { Desagregación } \\
\text { Competencia } \\
\text { Introducción del estilo de gestión privada }\end{array}$ \\
\hline
\end{tabular}

Fuente: Gallego (1996).

Las reformas aplicadas en el sector público desde la orientación de la NGP se basan, según Gallego (1996), en la desagregación de estructuras organizativas previamente integradas con el objetivo de impulsar la competencia entre ellas para acceder a más contratos de provisión de servicios; la competencia, a su vez, se origina a partir de mecanismos de promoción de la eficiencia. La secuencia temporal sería que organizaciones burocráticas son sometidas a mecanismos incentivadores de su eficiencia (reformas de los años ochenta), que, al no ser lograda, genera la reestructuración organizativa (desagregación) para promover la eficiencia al poner en competencia organizaciones del sector público con organizaciones del sector privado. Esta secuencia temporal representa un proceso en el que el control político mediante incentivos internos ha sido reemplazado por un control económico a través de un control externo.

Dos líneas de pensamiento se pueden identificar claramente en el desarrollo de la NGP y, por lo tanto, en las propuestas de reforma de los servicios públicos: por una parte, la teoría de public choice, orientada a restablecer el dominio del control político sobre la burocracia; por otra, las perspectivas managerialistas orientadas a restablecer el dominio de la gestión (la libertad para gestionar) sobre la burocracia. En el proceso de reforma de los servicios sanitarios en Cataluña, se puede identificar el proceso de desagregación como un proceso claramente impulsado, y también claramente logrado, en la medida en que el CatSalut se ha consolidado como un organismo autónomo que puede desarrollar fórmulas de gestión propias del sector privado a la hora de garantizar la provisión de servicios sanitarios. La reciente decisión de externalizar la gestión de los CAP sólo es un paso más en el proceso desagregador del ICS.

Por lo que respecta a la competencia, ésta ha sido incorporada a los servicios sanitarios de la Administración Pública, aunque sólo en la parte de la provisión de servicios — no así en la de la compra-, ya que se prevé la figura del CatSalut como organismo responsable de garantizar la cobertura de servicios sanitarios. La justificación, según Gallego (1996), es la voluntad de asegurar el control político sobre la gestión y los proveedores. 
La referencia al tercer elemento de esta dimensión es central en nuestro análisis, ya que alude a la introducción de mecanismos de promoción de la eficiencia en las diferentes organizaciones. En general, se puede decir que se considera políticamente como un elemento muy importante, pero su introducción ha sido muy lenta. Así, hasta 1995, la remuneración en función de resultados se había introducido en los contratos de gerentes de segunda línea, pero no en los de gerentes de región sanitaria; paralelamente, la descentralización de la capacidad de decisión sobre la gestión se está debatiendo, de manera que los gerentes de región sanitaria tienen libertad para gestionar, pero no tienen total autonomía con relación al presupuesto. De la misma forma, el presupuesto asignado al ICS ha sido traducido a términos contractuales y es obligatoria la facturación, aunque no hay penalización si las condiciones no se cumplen.

Por lo que respecta a la incentivación individual, un sistema de incentivos sobre los profesionales de la sanidad requeriría homogeneizar los salarios y las condiciones de empleo de la XHUP. Este punto está en proceso de concreción: en un primer momento, se ha logrado la elaboración de un convenio único para todos los centros que forman la XHUP. Como esto no garantiza condiciones de trabajo homogéneas, la acción sindical en estos centros se ha marcado el objetivo de hacerlo realidad.

Hemos analizado el proceso de gerencialización de los hospitales desde un enfoque centrado en el discurso de los actores implicados: direcciones de los hospitales, trabajadores y representantes sindicales. Lejos de querer realizar proyecciones hacia el futuro, nos hemos planteado interrogantes para conocer experiencias concretas de la introducción de herramientas gerenciales en la dirección de los hospitales. El estudio de estas herramientas gerenciales no se puede separar de las estrategias que ponen en marcha diferentes colectivos implicados; no se puede analizar al margen de los intereses de los actores, ya que correríamos el riesgo de presentar la gerencialización como un proceso inevitable sin ningún tipo de repercusión sobre los actores sociales.

El caso de los servicios sanitarios públicos tiene la peculiaridad de que forma parte del paquete de servicios que gestiona el Estado. Este hecho implica analizar el proceso de gerencialización contextualizándolo en unas determinadas coordenadas políticas y económicas, además de las estrictamente laborales. El proceso de gerencialización no puede verse como un proceso inevitable ni tampoco como un proceso uniforme en todos los casos. El análisis nos muestra que, si bien el contenido de los procesos gerencialistas es similar y equiparable en los tres casos estudiados, no lo son los efectos de estos procesos sobre las condiciones de trabajo. Por el contrario, se pueden constatar diferencias entre centros y, también, entre grupos de trabajadores.

Se constata una superposición de técnicas de gestión empresarial aplicadas a los hospitales: la gestión por competencias, la gestión por procesos, la dirección por objetivos, la gestión de la calidad total, etc. Desde nuestro punto de vista, esta superposición 
responde al propio desarrollo de las técnicas de gestión en el ámbito de la empresa, donde estas técnicas se ensayan en la medida en que la dirección tiene conocimiento de ellas. La implementación de estas prácticas de gestión forma parte de un programa más amplio de experimentación de técnicas que pueden conducir a la mejora de resultados en las organizaciones, siempre bajo los parámetros de lo que las direcciones entienden como eficacia y eficiencia en la gestión de recursos. Al igual que en las empresas privadas y, paradójicamente, de forma contraria a la propia teoría de estas prácticas de gestión, en los hospitales estudiados se han incorporado estos procedimientos con una escasa participación de los trabajadores, hecho que tiene consecuencias directas sobre la organización y las condiciones de trabajo de los mismos.

Los cambios organizativos se justifican, en última instancia, por parte de la gerencia de los hospitales, como un hecho inevitable fruto de las políticas de racionalización de los recursos basadas en la reducción del gasto. Desde nuestro punto de vista, esta argumentación se debe a cierto "fatalismo económico», por el cual toda acción estaría justificada por el hecho de hacer más con menos coste. Es un principio de racionalidad económica que siguen las empresas privadas y que se traslada a los hospitales que tratamos. No se trata de invalidar el principio en sí mismo, sino de ver cuáles son las razones objetivas de su aplicación y cuáles son las implicaciones que tiene sobre la organización del trabajo y las condiciones de trabajo de los trabajadores. Un principio «fuerte» de estas características, entendido como un "principio esencial», no puede ser discutido como base de los cambios organizativos, según el planteamiento gerencial, lo que define un tipo de estilo de liderazgo y dirección escasamente abierto a la participación de los trabajadores. Otra cosa es la manera de ponerlo en práctica.

En general, los sindicatos aceptan la necesidad de racionalizar el funcionamiento de la Administración Pública y, en concreto, de los hospitales públicos, sobre todo en lo que respecta a la calidad de los servicios, el control del gasto... Es decir, los sindicatos asumen como propios los objetivos de dar mejores servicios y controlar el gasto. Sin embargo, mantienen una postura muy crítica sobre las prácticas que se están implementando para hacer realidad estos objetivos, así como también sobre la forma de definir y poner en marcha estas prácticas: unilateralmente, por parte de las direcciones de los centros y sin negociar con los trabajadores ni sus representantes. En general, los sindicatos critican que el control del gasto esté centrado en la reducción de plantillas, ya que, a su entender, este elemento entra en contradicción con el objetivo de mejorar la calidad de los servicios. 


\section{Conclusiones: efectos de la gerencialización sobre las condiciones de trabajo}

En este análisis nos ha interesado conocer los efectos de las propuestas gerencialistas en relación con las condiciones de trabajo. Más allá de la descripción de los cambios, hemos querido ver si estos cambios garantizan o facilitan lo que políticamente se presenta como su justificación: la mejora final de los servicios. El análisis realizado nos permite destacar que no existe una determinación técnica de las medidas adoptadas en los casos estudiados y, por lo tanto, que estas herramientas de gestión no son artefactos neutros, no son únicamente técnicas de gestión, sino que tienen consecuencias en términos de condiciones de trabajo.

Se puede considerar que las condiciones de trabajo de los trabajadores públicos deben poder ser susceptibles de modificación, de la misma forma que las del resto de los trabajadores. Sin embargo, hay dos elementos que necesariamente ponen límites a esta consideración: la primera hace referencia a las repercusiones, generadas por los cambios en las condiciones de trabajo, sobre la calidad de los servicios finalmente ofrecidos; la otra hace referencia a la necesidad de tener en cuenta la especificidad del sector público.

La cuestión es, pues, cuál es el modelo de Administración que debe hacer frente a los cambios que está experimentando y, especialmente, cuál es el modelo de empleo que se debe dar en esta Administración. Esto no niega que haya empleos dentro de la Administración Pública que son equiparables a algunas realizadas fuera de ella. Sin embargo, hay tres elementos en la Administración que marcan la necesidad de una gestión diferenciada con respecto a la empresa privada: por una parte, el hecho de que los objetivos no están prefijados ni son unívocos; por otra, la necesidad de articular y conciliar intereses diversos entre colectivos y actores sociales; finalmente, la dimensión política de los servicios públicos, de manera que el conflicto no está centrado en el capital y el trabajo, sino en las características de estos mismos servicios, especialmente por lo que a la cantidad y la calidad se refiere.

En relación con la división y la organización del trabajo, se constatan cambios en la dirección de definir de manera más clara tanto el contenido del trabajo de cada grupo de trabajadores como las actuaciones que éstos deben realizar en función de la tipología de los pacientes; es la generalización de los protocolos de actuación. Estos cambios están generando efectos en términos de control sobre el trabajo. Por lo que respecta a los incrementos en la planificación del trabajo, la valoración de los facultativos es negativa, ya que reivindican más autonomía para decidir, por ejemplo, la cantidad de tiempo que requiere un paciente para ser visitado. Esto, sin embargo, choca con una planificación rigurosa del trabajo: cada tres minutos, un paciente. 
En otros aspectos, el colectivo de médicos considera que estos cambios no cuestionan su competencia; así, por ejemplo, viven la generalización de los protocolos de manera positiva, porque representan una guía para orientar su trabajo sin limitar su cualificación. Por su parte, el personal de enfermería considera que la generalización de la planificación de tareas y el aumento de protocolos inciden de manera negativa en su trabajo, ya que las obliga a un trabajo más rutinario y descualificado. La gerencia prevé el trabajo más fácilmente cuantificable: administrar tratamientos médicos; y se olvida del otro trabajo de enfermería, más cualitativo, sin embargo, en opinión de las enfermeras, tan importante como los tratamientos médicos: hablar con los enfermos, explicarles su situación, acompañar a las familias, etc.

En general, se constata que se da una preocupación, dentro de los programas gerenciales, por la cuantificación de tareas que conlleva, junto con la política de no incremento del gasto, un incremento de la carga de trabajo. Dos factores inciden de manera clara en este aumento: por una parte, cambios demográficos que generan un incremento notable de la demanda de servicios sanitarios; por otra, una política de control del gasto que busca incrementos de eficiencia económica sin incrementar la inversión en personal.

Según el trabajo de campo, a pesar de estos puntos de partida contradictorios, el sistema de servicios sanitarios se mantiene eficaz; es decir, resuelve los problemas médicos de la población. El elemento que hace posible la eficacia del sistema es el incremento de la carga de trabajo y la voluntariedad con que el personal facultativo intenta suplir los déficits de los servicios. Uno de los entrevistados ponía nombre a este proceso: es la técnica del "paciente padre», que consiste en que el médico trata al paciente, en casos de gravedad y cuando la saturación del sistema prevé unos plazos de realización de pruebas y tratamiento excesivamente largos en el tiempo, como si fuera su padre. De esta manera, el médico se implica personalmente en hablar con los responsables de los diferentes servicios que deben atender al paciente para que le reciban y comiencen el tratamiento sin respetar las pautas previstas por el sistema. Es decir, el sistema sanitario, a iniciativa de los profesionales, encuentra vías para no seguir los canales que el mismo sistema prevé. El efecto es que el sistema mantiene su eficacia (el paciente es finalmente tratado) gracias a la implicación de los facultativos; esto tiene efectos importantes sobre las condiciones de trabajo: por una parte, se incrementa la carga y la intensidad del trabajo; por otra, estas prácticas son de difícil cuantificación en términos de objetivos y resultados.

Los cambios en la jornada de trabajo y la remuneración también han generado una crítica muy importante por parte de los facultativos, sobre todo los médicos, que son el colectivo mayoritariamente afectado. Son los aspectos en los que la acción sindical, en los tres casos estudiados, se ha mostrado más activa. La jornada de trabajo está siendo 
revisada porque, desde los planteamientos economicistas de los programas gerenciales, se considera que la desaparición de las guardias y la introducción de trabajo por turnos podrían ser elementos que hiciesen posible el ahorro económico. En general, el colectivo de médicos está en contra del trabajo por turnos, porque considera que atenta contra su cualificación y competencia en la gestión de los pacientes. La retribución variable ha sido criticada, sobre todo, por la falta de control sobre los objetivos en atención a los cuales se cobrará.

Un elemento nuevo en la política de gestión de los trabajadores en todos los centros es el planteamiento de la carrera profesional. Este planteamiento intenta vincular los salarios de los colectivos de médicos y enfermeras con la obtención de mejoras en una serie de ítems previamente negociados. Este sistema es aceptado por los trabajadores como una herramienta de mejora salarial, pero, al mismo tiempo, es cuestionado en tanto que representa también una herramienta más de control sobre el trabajo del personal.

\section{Bibliografía}

Alba, C. R. (2000) «Desaparición o reestructuración del Estado: reformas de la Administración o reformas de la gobernación». En A. Trinidad (coord.), Evaluación y calidad en las organizaciones públicas. INAP: Madrid.

Albi, E. (2000): Público y privado: un acuerdo necesario, Ariel, Barcelona.

Albizu, E.; Olazaran, M. (2003): Reingeniería y cambio organizativo, PrenticeHall, Madrid.

Bañón Martínez, R. (1993): «La modernización de la administración pública española. Balance y perspectivas», Política y Sociedad, núm. 13.

Bañón Martínez, R. (1997): "Los enfoques para el estudio de la Administración Pública: orígenes y tendencias actuales», en R. BAÑón y E. CArrillo (comps.): La nueva Administración Pública, Alianza, Madrid.

Bañón, R.; Carrillo, E. (1997): "La legitimidad de la Administración Pública», en R. Bañon y E. Carrillo (comps.): La nueva Administración Pública, Alianza, Madrid.

Beltrán, M. (1991): La productividad de la Administración española: un análisis comparativo, Instituto de Estudios de Prospectiva, Madrid

Beltrán, M. (1991a): La realidad social, Tecnos, Madrid.

Beltrán, M. (1996)ः «Las Administraciones públicas», en J. Tusell et alii, (eds.): Entre dos siglos. Reflexiones sobre la democracia española, Alianza, Madrid. 
Beltrán, M. (1996a): «De la reforma de la Administración al control de calidad de los servicios públicos», Gestión y Análisis de Politicas Públicas, núm. 5-6.

Beltrán, M. (2000): La acción pública en el régimen democrático, Centro de Estudios Políticos y Constitucionales, Madrid.

Beltrán, M. (2000a): «El problema de la calidad en los servicios públicos», en A. Trinidad (coord.), Evaluación y calidad en las organizaciones públicas, INAP, Madrid.

Bozeman, B. (1998): "Introducción: dos conceptos de gestión pública», en B. BoZEMAN (coord.): La gestión pública: su situación actual, Colegio Nacional de Ciencias Políticas y Administración Pública, A. C., Universidad Autónoma de Tlaxcala, Fondo de Cultura Económica, México.

Bozeman, B. (1998a): "La teoría. La "sabiduría” y el carácter del conocimiento en la gestión pública: opinión crítica del nexo entre teoría y práctica», en B. BozemAN (coord.): La gestión pública: su situación actual, Colegio Nacional de Ciencias Políticas y Administración Pública, A. C., Universidad Autónoma de Tlaxcala, Fondo de Cultura Económica, México.

Brugué, Q; Amorós, M.; Gomà, R. (1994) «La Administración pública y sus clientes: ¿moda organizativa u opción ideológica?», Gestión y Administración de Politicas Públicas, núm. 1.

Brugué, Q.; Subirats, J. (1996), Lecturas de gestión pública, MAP, Madrid.

Brugué, Q. (1996): «La dimensión democrática de la nueva gestión pública», Gestión y Administración de Políticas Públicas, núm. 5-6.

Brugué, Q. (1998): Gestió pública i democràcia: de l'Administració del vídeo a l’Administració del xip, Diputació de Barcelona, Barcelona.

Fairbrother, P. (1994): «Un sector estatal en cambio: la flexibilidad en la administración pública», en A. Pollert (comp.): ¿Adiós a la flexibilidad?, Ministerio de Trabajo y Seguridad Social, Madrid.

Gallego Calderón” R. (1996): «Reformas de "New Public Management”: el caso de la Administración sanitaria catalana», Gestión y Administración de Politicas Públicas, núm. 5-6, pp. 163-174.

Gallo, P. (I998-I999): «Desigualdades en la provisión de servicios sanitarios», Gestión y Administración de Políticas Públicas, núm. 13-14.

Huertas, R. (1998): Neoliberalismo y politicas de salud, El Viejo Topo, Madrid.

Hughes, O. E. (1996): «La nueva gestión pública», en Q. Brugué y J. Subirats (comps.): Lecturas de gestión pública, Ministerio de Administraciones Públicas, Madrid.

Hyman, R. (1991): «Plus ça change? La teoría de la producción y la producción de la teoría», en A. Pollert (comp.): ¿Adiós a la flexibilidad?, Ministerio de Trabajo y Seguridad Social, Madrid. 
Metcalfe, L.; Richards, S. (1989): La modernización de la gestión pública, Ministerio de Administraciones Públicas, Madrid.

Metcalfe, L. (1996): "Gestión pública: de la imitación a la innovación», en Q. Brugué; J. Subirats (comps.): Lecturas de gestión pública, Ministerio de Administraciones Públicas, Madrid.

OlíAs de LimA, B. (1995): "Gestión pública, gestión privada de los recursos humanos», en B. Olías de lima (coord.): La gestión de recursos bumanos en las Administraciones Públicas, Complutense, Madrid.

Olías de lima, B. (coord.) (2001): La nueva gestión pública, Prentice Hall, Madrid.

Pollit, C. (1993): El «gerencialismo» y los servicios públicos. La experiencia anglonorteamericana, Instituto de Estudios Fiscales, Madrid.

Ramió, C.; Ballart, X. (1993): Lecturas de la teoría de la organización, vol. II, Ministerio de Administraciones Públicas, Madrid.

Ramió, C. (1999): Teoría de la organización y Administración Pública, Tecnos, Madrid

Villoria, M. (1996): La modernización de la Administración como instrumento al servicio de la democracia, Ministerio de Administraciones Públicas, Madrid. 